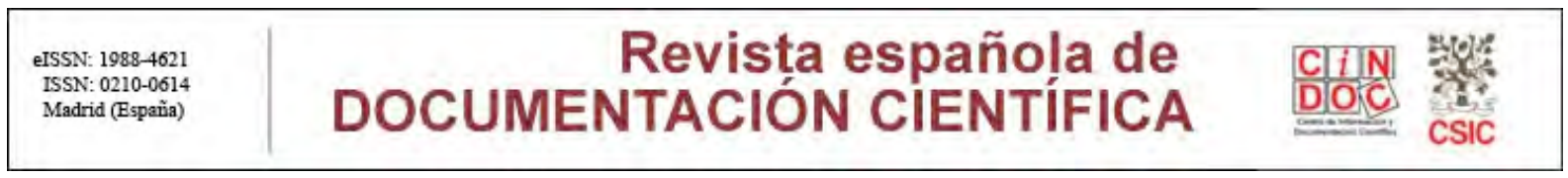

Revista Española de Documentación Científica [online]

ISSN: 0210-0614

eISSN 1988-4621

\title{
Retractación
}

La Revista Española de Documentación Científica, en aplicación de las directrices marcadas por los comités internacionales de ética editorial, y las normas de buenas prácticas en la publicación científica, sólo admite artículos originales e inéditos, es decir, no publicados previamente total o parcialmente en una o varias revistas, tanto en versión impresa como electrónica. Tampoco acepta los trabajos que se hayan enviado o estén en proceso de evaluación para su publicación en otra revista.

Por tanto, la dirección de la Revista Española de Documentación Científica ha decidido proceder a la retractación formal del siguiente trabajo:

Moreno-Cabo, M. y Solás-Portolés, J.J. Estudio bibliométrico de las publicaciones relacionadas con el péndulo entre los años 1629 y 1885. Revista Española de Documentación Cientifica, 2008, vol. 31, n 4, p. 639-645. doi: 10.3989/redc.2008.4.647

Ya que se trata de una duplicación exacta (salvo tres párrafos de la introducción) de otro publicado previamente:

Solas-Portolés, J.J.; Moreno Cabo, M.; Sanjosé López, V. Aprendiendo cómo se construye la ciencia: el caso del péndulo. Latin American Journal of Physics Education, 2008, vol. 2, n ${ }^{\mathrm{o}}$, p.47-50.

El trabajo se envió casi simultáneamente a ambas revistas. En la REDC se recibió en el 20 de noviembre de 2007, y se publicó en el último número del año 2008 (octubre-diciembre), y en LAJPE, se recibió el 12 de diciembre de 2007, y se publicó el 9 de enero de 2008.

En ninguno de los trabajos se hace referencia al otro, ni se indican las razones que estimaron los autores para enviar un duplicado a dos revistas diferentes. Además, el hecho de haber cambiado el título, el orden de los autores, y haber incluido un tercer autor en el primer trabajo que se publicó, no puede ocultar el contenido idéntico de ambos artículos.

Esta nota editorial se incorpora a la versión electrónica del artículo y se publicará en el próximo número impreso de la Revista Española de Documentación Científica, v. $34, \mathrm{n}^{\circ} 1$, de 2011.

Madrid, 26 de enero de 2011. 
NOTAS Y EXPERIENCIAS / NOTES ANDEXPERIENCES

\section{Estudio bibliométrico de las publicaciones relacionadas con el péndulo entre los años 1629 y 1885}

\section{A Bibliometric study on the publications related to the pendulum between} 1629 and 1885

\section{Magdalena Moreno-Cabo*, Joan Josep Solaz-Portolés**}

Resumen: En este trâbajo se lleva a cabo un análisis bibliométrico de una bibliografía de trabajos relacionados con el péndulo. Se determinan indicadores de la actividad científica, en concreto, número de publicaciones, productividad de los autores y de colaboración en las publicâciones. Además, se evalúa el porcentaje de trabajos en las lenguas mayoritarias (Francés, Latín, Italiano, Inglés y Alemán). De los rêsultados obtenidos se concluye que: a) El crecimiento del número de trabajos en el período considerado es exponencial; b) La productividad de los autores no sigue la ley de Lotka; c) ET índice de firmás por trabajo es muy bajo $(1,1)$; d) El Francés es la única lengua que se mantiene hegemónica prácticamente durante todo el período 1629-1885; e) El Latín, muy potente hasta finales del XVIII, desaparece en el siglo XIX coincidiendo con el espectacular crecimiento del Inglés yel Alemán. La evolución dé las lenguas de los trabajos se analiza en función del contexto histórico en los correspondientes países.

Palabras clave: péndulo, bibliografía, bibliometría, número de publicaciones, productividad de autores, firmas por trabajo, lenguas de los trabajos.

Abstract: This paper reports a bibliometric analysis of a bibliography of works on the pendulum. Indicators of scientific activity were determined, including number of publications, author productivity, and collaborations. In addition, the percentages of works in the different majority languages (French, Latin, Italian, English and German) were calculated. The results show that: a) the growth of the number of works in the analysed period was exponential; b) author productivity did not follow Lotka's law; c) the number of signing authors per work is very low (index 1.1); c) French retained its linguistic hegemony for almost the entire period examined (1629$1885)$; d) Latin, which was very powerfully represented until the end of the

* Biblioteca P.M. de L'Eliana. L’Eliana. Valencia. Correo-e: malena.5@hotmail.com.

** IES «F. Tomás y Valiente». UNED. Benaguasil. Valencia. Correo-e: jjsolaz@ valencia.uned.es. Recibido: 20-11-07; 2. ${ }^{a}$ versión: 11-5-08. 
$18^{\text {th }}$ century, disappeared in the $19^{\text {th }}$ century, coinciding with the spectacular growth of English and German. The change in the languages employed in these works is analysed with respect to the historical contexts of the corresponding countries.

Keywords: pendulum, bibliography, bibliometrics, number of publications, author productivity, collaboration in publication, number of signing authors per work, languages of publication.

\section{Introducción}

El péndulo, un humilde instrumento, ejerció ûna notẩble influencia en el desarrollo conceptual de la ciencia mecánica, contribuyendo a conformar uno de los pilares de la Física clásica: el paradigma Newtôniano (Solaz-Portolés y Sanjosé, 1992). De acuerdo con Matthews, Gauld y Stinner (2005), el péndulo fue crucial para establecer, entre otras cosas, la ley de colisiones, las leyes de conservación, el valor de la aceleración de la gravedad, la variación de la aceleración de la gravedad con la latitud terrestre, y probablemente, proporcionó a Newton los indicios para que formulara su síntesis de la mecánica celeste y terrestre. Hablamos genéricamente de péndulo, no obstante, a to largo de la historia se han construido diferentes tipos de ellos y con distintas aplicaciones técnicas (Baker y Blackbum, 2005).

Para hacernos una idea de la ímportancia instrumental que tuvo el péndulo en la física y otras ciencias, baste con señalar que el numero de referencias bibliográficas sốbre su teoría y aplícaciones citadas en Bibliographie du pendule por Wolf (1889a), asciende aproximadamente a unas mil trescientas en el período comprendido entre el año de publicación en Florencia de la obra de Galileo Dialogo intor no ai due massimi sistemi del mondo, Tolemaico e Copernicano y 1885. Indicaremos, en relación con estos trabajos publicados, que en ellos aparecen científicos tan sobresalientes como: Galileo, Huygens, Newton, Hooke, Halley, Mariotte, Bernoui1li, Celsius, D'Alambert, Poisson, Foucault, Stokes, etc.

Como deja entrever Khun (1987) Galileo fue el descubridor del péndulo. Parece claro que mucho antes del nacimiento de Galileo muchas personas habían visto oscilar un objeto del extremo de una cuerda hasta que finalmente quedaba en reposo. Sin embargo, Galileo rompió con el paradigma aristotélico y vio el péndulo como un cuerpo que seguía un movimiento periódico. A partir de ese momento, el péndulo tuvo un sinnúmero de aplicaciones en la ciencia y en la técnica (Wolf 1889b).

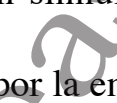

Justamente por la enorme importancia que ha tenido el péndulo en el desarrollo de la ciencia, consideramos interesante la realización de un estudio bibliométrico del conjunto de publicaciones proporcionadas por Wolf (1889a) en su Bibliographie du pendule. Este estudio pretende mostrar un caso particular -el péndulo y sus aplicaciones científico-técnicas- del modo de crecimiento de la ciencia en el período com- 
prendido entre 1629 y 1885 , así como ciertos aspectos de la forma en que sus actores trabajaban: productividad de los autores, colaboración en los trabajos y lenguas que utilizaban. Intentaremos, también, siquiera sea brevemente, establecer las oportunas correspondencias con la realidad social del momento en los distintos países.

\section{Metodología}

Dispusimos para nuestro trabajo de ûna fuente secundaria consultada en la Biblioteca de las Facultades de Ciencias de la Universitat de València. Esta fuente secundaria es el Tomo IV de la Collection de Mémoires relatif's a la Physique, publicadas por La Societé Françaisé de Physique. Este tomo IV, Mémoires sur le pendule, contiene una introducción histórica y una bibliografî́a de trabajos sobre el péndulo. La bibliografía recoge artículos, libros, informes de instituciones científicas y disertaciones; y proporciona el nombre de los autores, el título del trabajo y un breve resumen del mismo. Hemos de resaltarque es la únicá fuente de estas características que hemos podido encontrar que cubra el período de tiempo en el que estamos interesados: siglos XVII al XIX. Presumimos que es la única y además muy exhaustiva. Uno de los investigadores que más ha profundizado en la historia del péndulo, Michael R. Matthews, no cita esta fuente en el listado de referencias bibliográficas que ofrece en su sitio web (Matthews, 2008). Por otro lado, hemos comprobado que recoge todos aquellos trabajos que han supuesto hitos en la historia de las física, y también otros de gran interés para la cartografía, geografía, mineralogía, etc.

Se determinaron indicadores de la actividades científica, en concreto, número de publicaciones, productividad de los autores y de colaboración en las publicaciones (Sancho, 1990). Además, se realizó una búsqueda de las lenguas mayoritarias en los trabajos. Para ello, se llevó a cabo el cómputo de publicaciones por períodos de diez años, se contabilizó los distintos trabajos que publicó cada autor, y se calculó el porcentaje de trabajos en las lenguas mayoritarias en períodos de veinte años.

\section{Resultados}

La figura 1 recoge la representación gráfica de la suma de trabajos publicados en relación con el péndúlo en períodos de tiempo de diez años (el primer período sólo incluye siete años, desde 1629 hasta 1635).

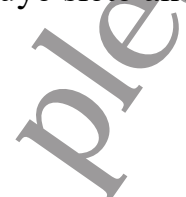


Figura 1

Representación gráfica del número de publicaciones relacionadas con el péndulo contadas en períodos de diez años

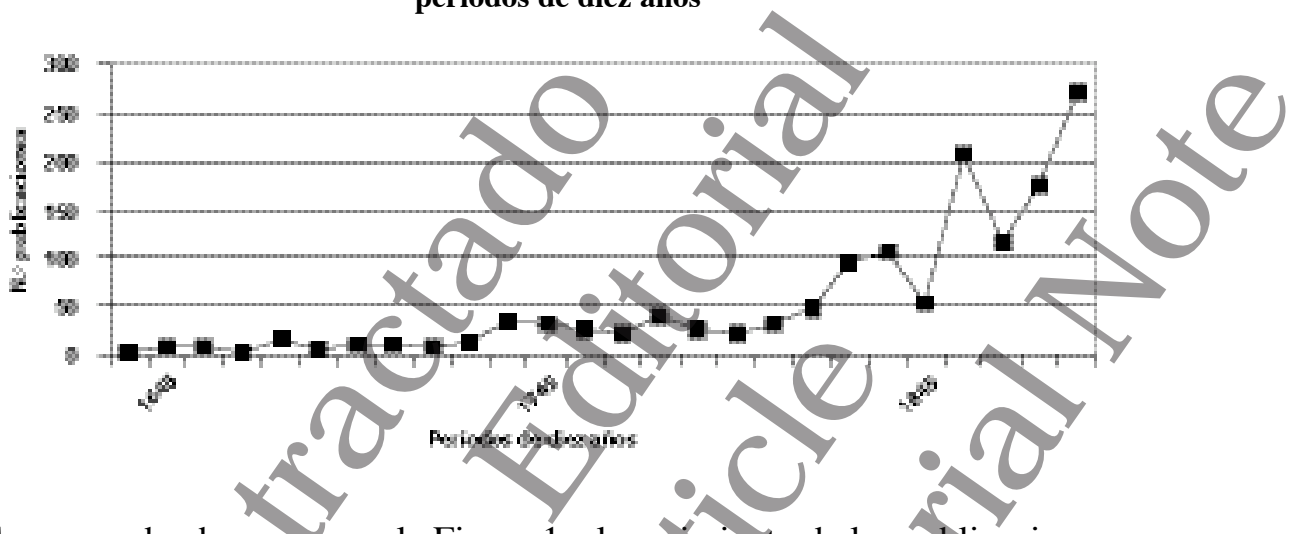

Como puede observarse en la Figura 1, el crecimiento de las publicaciones parece ajustarse a una función exponencial. De hêcho, el análisis de regresión lineal entre el logaritmo del numero de publicaciones y el períodó de tiempo, nos proporciona un coeficiente de correlación lineal $r=0.93$, valor que viene a confirmar el carácter exponencial de la relación entre el numero de publicaciones y el tiempo entre los años 1629 y 1885 .

La Figura 2 representa lá distribución del número de autores según el número de publicaciones relacionadas con el péndulo. La curva establece el número de $y$ autores que han publicado $x$ trabajos.

Representación gráfica del número de autores en función del número de trabajos que publican

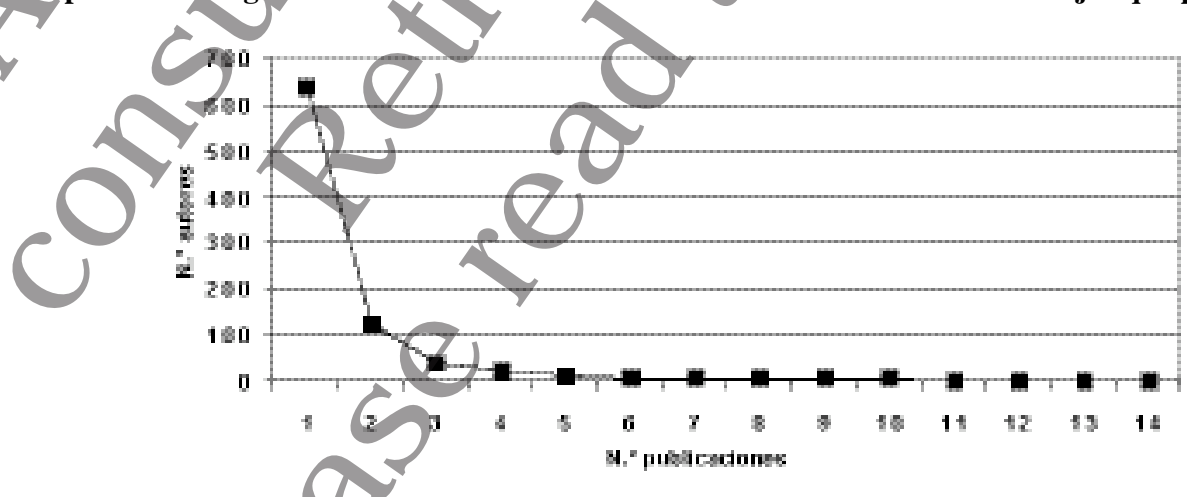

Se constata, a partir de la Figura 2, que sólo un pequeñísimo número de autores publicó en el período de tiempo considerado más de cinco trabajos relacionados con el péndulo, mientras que más de seiscientos no publicaron más que un único trabajo. Por otra parte, el análisis de regresión lineal entre el logaritmo del número de autores y el logaritmo del número de publicaciones, nos proporciona un coeficiente 
de correlación lineal $r=0.998$ y una pendiente de la recta de $-11,43$. Por tanto, la curva se ajusta de manera aproximada a la ecuación $y=k / x^{11}$.

Por otra parte, el cociente entre el número total de publicaciones entre 1629 y 1885 (1324 trabajos) y el número total de autores (1388), nos permite obtener el índice de firmas por trabajo, que resulta ser de 1,1 .

Finalmente, la Figura 3 nos proporciona el porcentaje de publicaciones en cada una de las cinco lenguas más utilizadas (francés, italiano, latín, inglés y alemán) en períodos de veinte años (el primer período sólo incluye diecisiete años, deșde 1629 hasta 1645).

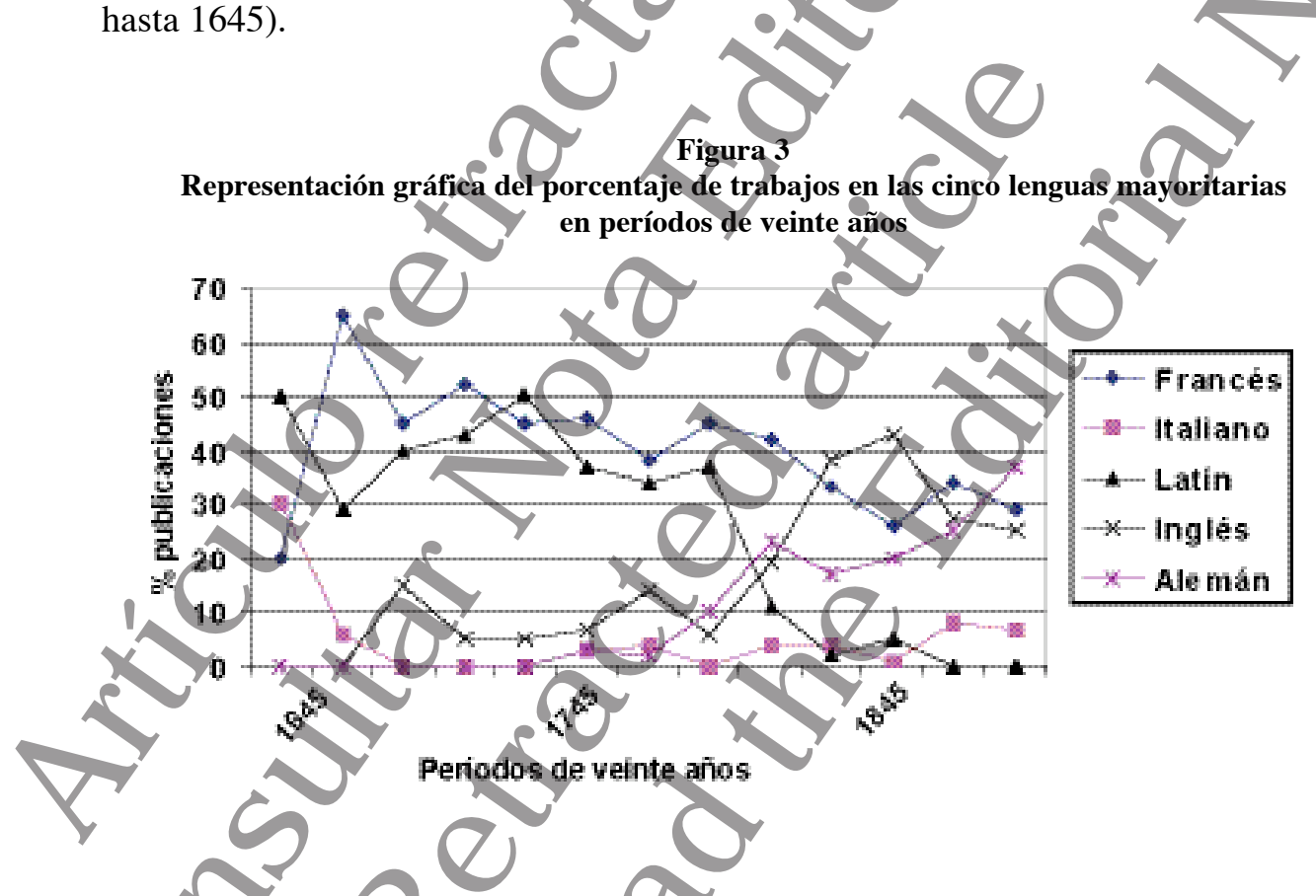

Varias circunstancias son destacables de esta última representación gráfica. En primer lugar, el latín, de ser una lengua de comunicación científica de primer orden -como el francés- hasta 1785 , pasa prácticamente a desaparecer en la segunda mitad del siglo XIX. El inglés, minoritario hasta finales del siglo XVIII, se convierte en una de las tres lenguas de la ciencia en el siglo XIX. El alemán, no utilizado hasta la primera mitad del siglo XVIII, se transforma en la lengua de la Física en el final del siglo XIX. El francés, es la única lengua que se mantiene como mayoritaria durante todo el período de tiempo considerado. El italiano, de ser lengua mayoritaria en los primeros años de publicaciones sobre el péndulo, evoluciona posteriormente a minoritaria. Por último, señalaremos que el español, que no figura en esta representación gráfica por ser lengua muy minoritaria, aparece en la primera mitad del siglo XVIII (un trabajo) y, a lo largo de los años computados del siglo XIX, contabilizamos un total de cinco trabajos. 


\section{Conclusiones y discusión}

El crecimiento exponencial de las publicaciones a lo largo del período de tiempo que se ha tenido en cuenta en el presente trabajo, revela el dinamismo de las áreas de conocimiento relacionadas con el péndulo. En consecuencia, parece cumplirse en este período la ley normal del crecimiento de la ciencia propuesta por Solla Price (1973). Sin embargo, no podemos dejar de apuntar que este modo de crecimiento de la ciencia no puede servir de apoyo a una concepción acumulativa de la ciencia (Hragh, 1989). Como denuncia T. S. Khun (1987) existe una tendencia persistente a hacer que la historia de la ciéncia parezca lineal o acumulativa, obviándose las rupturas o cambios profundos que se producen en toda ciencia.

Resulta destacable comentar que el número de trabajos comienza a crecer de manera importante a finales del siglo XvII, época que coincide con una gran difusión de la ciencia y de ingreso de crecientes masas de jóvenes en centros de instrucción superior, Esto tuvo como consecuencia la divî́sión de trabajo entre los científicos y la especialización en las distintas ramas del saber, lo que favoreció el aumento de nuevos descubrimientos en cada campo de investigaciones (Geymonat, 1985a).

En cuanto a la productividad de los autores, de los resultados del presente trabajo puede concluirse que no se cumple en este casola ley cuadrática inversa ( $y=$ $k / x^{2}$ ) o ley de Lotka (Callon, Courtial y Penan, 1995). La curva obtenida para el decrecimiento delnúmero de autores en función del número de trabajos que publica se ajusta mejor a la ecuación $y=k / x^{11}$. Esto es, tiene un decrecimiento mucho más rápido (superiớ a cinco veces más rápido) que el previsto por Lotka en su ley. Esta desviación puede justificarse atendiendo, sobre todo, al elevadísimo número de autores que sólo publica un trabajo. Esste hecho, también viene reflejado en el bajísimo índice de firmas por trabajo $(1,1)$, que contrasta con la media de firmas por trabajo que se registra en la actualidad para las ciencias: entre 2,5 y 3,5 (Sancho, 1990).

El crecimiento del número de trabajos relacionados con el péndulo en lengua francesa se corresponde, asimismo, con los comienzos de la ilustración y el progreso científico que se produce en los albores del siglo XVIII (Geymonat, 1985b), y que posteriormente conduce a la primacía de Francia en el campo de la investigación científica en el período que sigue a la Revolución Francesa (entre los siglos XVIII y XIX). Como apunta Bernal (1979, p. 389) «la Revolución francesa y las guerras napoleónicas no provocaron un descenso de la actividad científica, sino más bien un impulso considerable de la misma».

Por otra parte, eldescenso del porcentaje de publicaciones en latín en el inicio del siglo XIX, es coincidente con el auge de publicaciones en inglés y alemán. Sobre este punto, cabe señalar que en la primera mitad del siglo XIX se produce la rápida difusión a Inglaterra y Alemania del espíritu técnico-científico, que había singularizado en exclusiya a Francia, y que iba indisolublemente ligado al proceso de indus- 
trialización de estos países (Geymonat, 1985a). Por tanto, no debe resultarnos nada extraño que ambos países llegaran a ocupar puestos de primer orden en el área de conocimientos de la Física en la segunda mitad de dicho siglo, dado que se atrajo a la investigación científica a muchos jóvenes.

\section{Bibliografía}

Baker, G.L.; Blackbum, J.A. (2005). The pendulum. A case study in physics. Oxford; Oxford University Press.

Bernal, J. D. (1979). Historia social de la ciencia, I. La ciencia en la historia, 5. ed., Barcelona Ediciones Península, p. 389.

Callon, M.; Courtial, J. P.; Penan, H. (1995). Cienciometria. La medición de la actividad científica: de la bibliometría a la vigilanciá tecnológica. Gijón; Edieiones Trea, p. 44.

Geymonat, L. (1985a). Historia de la filosofia y de la ciencia. T. 3 El pensamiento contem poráneo. Barcelona, Editorial Crítica, pp.110 y 178.

Geymonat, L. (1985b). Historia de la filosofía y de la ciencia. T.2 Del renacimiento a la ilustración. Barcelona, Editorial Crítica, p.261.

Kragh, H. (1989). Introducción a la historia de la ciencia. Barcelona, Editorial Crítica, p. 243.

Kuhn, T. S. (1987). La estructura de las revoluciones científicas (11 ${ }^{\text {a }}$ reimpresión). Madrid, Ediciones FCE, pp 187 y 216.

Matthews, M.R. (2008). Pendulum. A project of the School of Education. New South Wales:

1 Faculty of Arts and Social Sciences, University of New South Wales. Recuperado 7

mayo 2008 de http://www.arts.unsw:edu.au/pendulum/about.html

Matthews, M.R.; Gauld, C.; Stinner, A. (2005). The pendulum: Its place in Science, Culture and Pedagogy. En: M.R. Matthews, A. Stinner, C.F. Gauld (eds.), The pendulum.

Scientific, Historical, Philosophical and Educational Perspectives. Dordrecht, Springer, pp. $1-17$.

Sancho, R. (1990). Indieadores bibliométricos utilizados en la evaluación de la ciencia y la tecnología. Revista Española de Documentación Científica, vol. 13 (3-4), 842-865.

Solaz-Portolés, J. J.; Sanjosé, V. (1992). El papel del péndulo en la construcción del paradigma newtoniano. Enseñanza de las ciencias, vol. 10 (1), 95-100.

Solla Price, D.J. (1973). Hacia ûna ciencia de la ciencia, Barcelona, Editorial Ariel, p. 55.

Wolf, C. (1889a). Bibliographie du pendule. En: C. WOLF (Editor), Collection de mémoi res relatifs a la Physique, publiés par la Société Française de Physique. Tome IV Mémoires sur le pendule. Gauthiers-Villars et Fils, París, France.

Wolf, C. (1889b). Introduction historique. En: C. WOLF (ed.), Collection de mémoires rela tifs a la Physique, publiés par la Société Française de Physique. Tome IV Mémoires sur le pendule. Gauthiers-Villars et Fils, París, France.

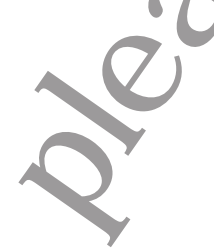

